\title{
Overexpression of truncated ERG from TMPRSS2-ERG fusion and prostate cancer development
}

This article was published in the following Dove Press journal:

Pathology and Laboratory Medicine International

29 September 2009

Number of times this article has been viewed

\author{
Melanie Leong ${ }^{*}$ \\ Wen-feng Shi ${ }^{2 *}$ \\ Jun Tian ${ }^{2}$ \\ Ellen Cho' \\ Abbas Raza' \\ Saquib A Siddiqi' \\ Abdulhafez Selim ${ }^{3}$ \\ Han-chun Chen ${ }^{4}$ \\ Dianzheng Zhang ${ }^{1,4}$ \\ 'Department of Biochemistry \\ and Molecular Biology and Center \\ for Chronic Disorders of Aging, \\ Philadelphia College of Osteopathic \\ Medicine, Philadelphia, PA, USA; \\ 2Department of Renal Transplantation, \\ Qilu Hospital of Shandong University, \\ Jinan, Shandong, People's Republic \\ of China; ${ }^{3}$ Osteotech Inc, Eatontown, \\ NJ, USA; ${ }^{4}$ Department of Biochemistry, \\ School of Biological Science and \\ Technology, Central South University, \\ Changsha, Hunan, People's Republic \\ of China; *These authors contributed \\ equally to this work
}

\begin{abstract}
In men, prostate cancer is one of the most common cancers worldwide and the second leading cause of death among all cancer types in Europe and North America, with the numbers of those affected continuing to increase. Recent studies have identified a recurrent fusion of TMPRSS2 with members of the ETS family of transcription factors in about $80 \%$ of prostate cancer tissues. Among them, the TMPRSS2-ERG fusion accounts for approximately $50 \%$ of these cases. TMPRSS 2 is highly regulated by androgen receptor and the chromosomal rearrangement abnormally induces ERG production by androgen. To investigate the effects of ERG overexpression on its target genes expression and prostate cancer development, plasmids were first constructed by inserting the truncated $E R G$ into an expression vector in the forward or reverse directions. A predicted three-dimensional model of the protein structure of the truncated ERG, along with immunofluorescence assays, suggest that the minor deletion on the N-terminus does not appear to affect the structure or function of ERG. Results from ERG target gene expression profile indicate that TMPRSS2-ERG fusion-induced aberrant ERG overexpression is likely involved in prostate cancer development by enhancing tumor angiogenesis.
\end{abstract}

Keywords: prostate cancer, androgen receptor, TMPRSS2, ERG, chromosomal translocation

\section{Introduction}

Prostate cancer is one of the most prevalent threats to men's health in the Western world and it accounts for the second largest number of male cancer deaths in the United States. ${ }^{1-5}$ It is understood to be a disease of complex and multifactorial origins. It appears that race, family history, and age are major factors related to its incidence. Men who have first-degree relatives with prostate cancer are at increased risk for the disease, with the risk of developing prostate cancer increasing with the number of relatives diagnosed. ${ }^{6}$ Males under the age of 50 have low rates of occurrence. However, rates increase rapidly after the age of 50 years, with 73 years being the average age at time of diagnosis., ${ }^{2,7}$ Because of improvements in the standards of modern living and advances in medicine, life expectancy has progressively increased and as a result, prostate cancer incidences are expected to increase proportionally. Hormone therapy benefits about $80 \%$ of prostate cancer patients by retarding the progression of the disease ${ }^{8}$ However, prostate cancer in the majority of the patients who initially benefited from hormonal therapy will eventually develop into an aggressive, hormone-independent form, with little hope for further intervention. ${ }^{9}$ Despite the high prevalence of prostate cancer, knowledge about the molecular and cellular mechanisms that underlie the cause of the disease is limited, and no cure yet exists for late stage prostate cancer.
Correspondence: Dianzheng Zhang Department of Biochemistry and Molecular Biology and Center for Chronic Disorders of Aging, Philadelphia College of Osteopathic Medicine, Philadelphia, PA, I9I3I, USA

$\mathrm{Tel}+|21587| 6588$

Fax + I $21587 \mid 6865$

Email dianzhengzh@pcom.edu 
The recently identified high incidence recurrent fusion of TMPRSS2 with members of the E26 transformation-specific (ETS) family of transcription factors, including $E R G, E T V 1$, ETV4, and ETV5, in prostate cancer samples has opened a new field in prostate cancer research. ${ }^{10-12}$ This is significant not only for understanding the underlying molecular mechanisms of prostate cancer development, but also for designing strategies for diagnosis, prognosis, and the differential treatment of prostate cancer. ${ }^{10,13,14}$ About $80 \%$ of prostate tumors contain genomic fusions of TMPRSS2 and members of the ETS family of transcription factors. Of these, $\sim 50 \%$ contains TMPRSS2-ERG fusions. ${ }^{15,16}$ Furthermore, it has been reported that TMPRSS2-ERG-positive prostate cancers have more aggressive phenotypes than those of TMPRSS2-ERGnegative cancers. ${ }^{17,18}$ Consequently, both short-term and long-term survival rates of patients with such fusions are significantly affected. ${ }^{19}$ Screening of prostate cancer samples found that among the 27 ETS factors, ERG is the most frequently overexpressed..$^{20}$ The TMPRSS 2 gene is located on chromosome 21 at 21q22.3 (Figure 1A) and encodes for a type II transmembrane serine protease. ${ }^{21}$ Normal prostate cells express little to no detectable TMPRSS2 protein, but high levels of TMPRSS2 protein have been found in a large percentage of prostate cancer cells. ${ }^{22} \mathrm{~A}$ recent study has demonstrated that the full-length TMPRSS2 protein is expressed in LNCaP cells, an androgen-responsive prostate cancer cell line, but is not detected in the androgenunresponsive prostate cancer cells PC-3 and DU-145. ${ }^{21}$ The expression of TMPRSS2 quickly increases in $\mathrm{LNCaP}$ cells after exposure to androgenic hormones indicating that it is likely a primary androgen receptor (AR) target gene. ${ }^{21}$ In fact, the TMPRSS2 promoter region has a 15 -bp sequence that
A

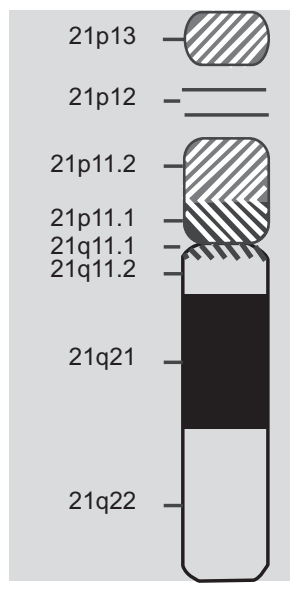

Chromosome 21

\section{B}

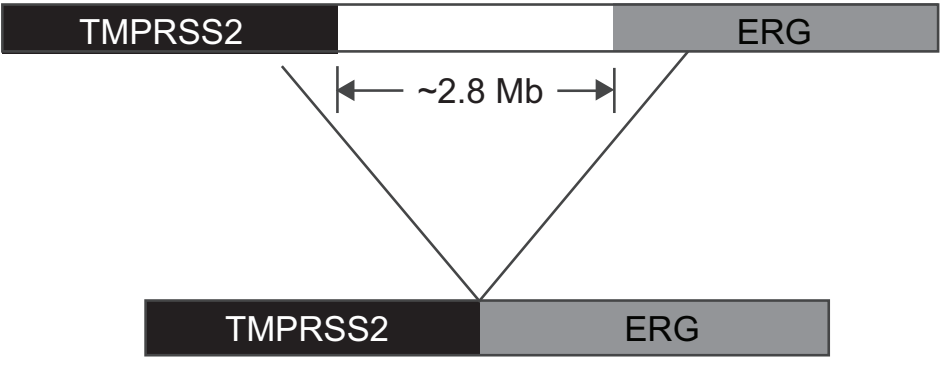

TMPRSS2-ERG gene fusion

C

TMPRSS2

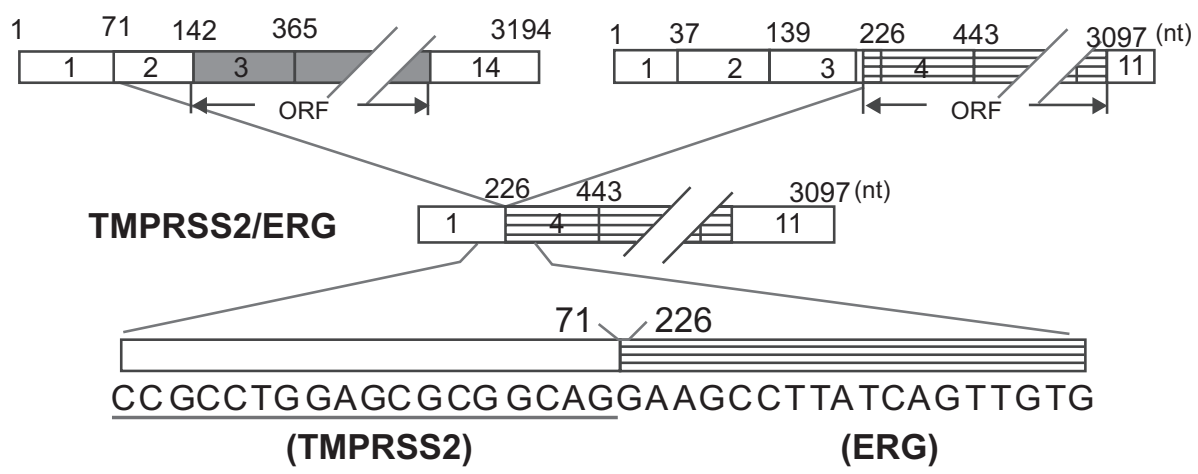

Figure I Chromosomal deletion and TMPRSS2-ERG fusion. A) Ideogram of chromosomal 2I. B) Interstitial deletion and formation of the TMPRSS2-ERG fusion. C) Schematic illustration of the exons and gene sequences in the TMPRSS2-ERG gene fusion. The gray box represents the open reading frame of TMPRSS2 while the striped box represents the open reading frame of ERG. Each exon is indicated by numbers in the boxes and the numbers above the boxes are the last base of each exon. Sequences at the fusion junction are indicated and the sequence from TMPRSS2 is underlined. 
contains a consensus AR-responsive element (ARE) which is likely involved in AR-ARE interaction and in the regulation of TMPRSS2 expression. ${ }^{23}$ Previous experiments have also suggested that the androgen-responsive promoter elements of TMPRSS 2 are implicated in prostate cancer through the overexpression of members of the E26 transformationspecific (ETS) family. ${ }^{19}$

One effect of the interstitial $3 \mathrm{Mb}$ deletion from chromosomal region $21 \mathrm{q} 22$ is the generation of the TMPRSS2-ERG fusion gene, which contains the promoter/enhancer region of the TMPRSS 2 gene and a part of the open reading frame of the ERG gene (Figure 1B). There are at least 17 distinct TMPRSS2-ERG transcripts generated from fusions between exons 1, 2, and 3 of TMPRSS 2 and exons 2, 3, 4, 5, or 6 of $E R G$ which have been identified, ${ }^{24}$ with the most commonly identified fusion in prostate cancer containing the promoter/ enhancer region and first exon of TMPRSS 2 gene and exon 4 of $E R G$ gene (Figure 1C). ERG is known to be a pivotal transcription factor in cell differentiation and growth control pathways, and there are lines of evidence to suggest that overexpression of ERG may be involved in different stage of prostate cancer development. ${ }^{13,14,16,25}$ However, the molecular mechanisms underlying the chromosomal translocation and prostate cancer development are not known. ${ }^{20}$ We hypothesize that overexpression of the ETS factors resulting from TMPRSS2-ETS fusion plays an essential role in prostate cancer development, and the data from our research suggest that overexpression of the truncated ERG may affect prostate cancer development, most likely through enhancing angiogenesis.

\section{Materials and methods Construction of overexpression vector, PSG5-ERG}

Total RNA was purified from the $\mathrm{VCaP}$ prostate cancer cell line using an RNAqueous-4PCR kit (Ambion, Inc., Austin, TX). For cDNA synthesis, $5 \mu \mathrm{g}$ of total RNA was reverse transcribed using random primers (Stratagene, La Jolla, CA) and SuperScript II reverse transcriptase (Invitrogen, Carlsbad, CA). The truncated open reading frame (ORF) of $E R G$ was amplified by PCR using the following primers: Upper primer, 5'-ATGACCGCGTCCTCCTCCAGCGA-3'; Lower primer, 5'-TTAGTAGTAAGTGCCCAGATGAG-3'. XhoI and NheI restriction enzyme sites were added to the upper and lower primer ends, respectively. The full length of the ORF in the fusion gene was approximately $1.2 \mathrm{~kb}$, and the amplified PCR product was purified with a Qiagen Gel Extraction kit (Qiagen, Inc., Valencia, CA). The PCR product was cloned into a TOPO-TA vector (Invitrogen). ERG was then sub-cloned into the PSG5 expression vector (J. Wong lab, Baylor College of Medicine, Houston, TX) on the XhoI site using T4 DNA ligase (Promega, Madison, WI). A negative control was constructed with the insert oriented in the opposite direction. Orientations were determined by digesting with NheI (Promega). DNA sequence and cloning junction were verified by sequencing (University of Pennsylvania DNA Sequencing Facility, Philadelphia, PA).

\section{Cell culture and transient transfection}

HeLa cells were maintained in Dulbecco's modified Eagle's medium (DMEM) (Cellgro, Mediatech, Inc., Herndon, VA) containing 10\% fetal bovine serum (FBS) (Atlanta Biologicals, Lawrenceville, GA) and 1\% antibiotics/antimycotics (Abx/Amx) (Gibco, Carlsbad, CA). NIH 3T3 cells were maintained in DMEM (Cellgro, Mediatech, Inc.) containing $10 \%$ bovine calf serum (BCS) (Atlanta Biologicals, Lawrenceville, GA) and 1\% Abx/Amx (Gibco). Cells were incubated at $37{ }^{\circ} \mathrm{C}$ and $4.7 \% \mathrm{CO}_{2}$. HeLa and NIH $3 \mathrm{~T} 3$ cells were transfected with plasmids containing either the forward insert or the reverse insert, using lipofectamine and PLUS reagent (Invitrogen). Cells were incubated in reduced serum opti-modified Eagle's medium (Opti-MEM) (Gibco) for six hours. After this six-hour incubation, DMEM containing 1\% Abx/Amx and either $10 \%$ FBS or $10 \%$ BCS was added to the $\mathrm{HeLa}$ and NIH $3 \mathrm{~T} 3$ cells, respectively. Cells were incubated for an additional 36 hours at $37^{\circ} \mathrm{C}$ and $4.7 \% \mathrm{CO}_{2}$.

\section{Lysis and western blotting}

Cells were collected and lysed with EBC buffer containing $20 \mathrm{mM}$ Tris $\mathrm{HCl}$ (pH 8.0), 125 mM EDTA, and 0.5\% NP-40. Protein samples were separated by SDS-PAGE electrophoresis and transferred to a nitrocellulose membrane. All membranes were stained with Ponceau S Solution to confirm successful transfer of the proteins (Sigma-Aldrich, St. Louis, MO). They were then blocked with 5\% milk in TBST (Tris-base, $\mathrm{NaCl}$, and 1\% Tween 20, pH 7.6). Proteins were incubated in $2.5 \%$ milk in TBST with ERG-1/2/3 rabbit polyclonal IgG $(0.2 \mu \mathrm{g} / \mathrm{mL})$ (Santa Cruz Biotechnology, Santa Cruz, CA) as a primary antibody and donkey-anti-rabbit $\operatorname{IgG}(0.08 \mu \mathrm{g} / \mathrm{mL})$ (Pierce Biotechnology, Rockford, IL) as a secondary antibody. The blot was stripped and reprobed by anti-actin $(1 \mu \mathrm{g} / \mathrm{mL})$ (Calbiochem, San Diego, CA) and peroxidase goat antimouse $\operatorname{IgM}(0.01 \mu \mathrm{g} / \mathrm{mL})$ (Calbiochem). The blots were detected using the Super Signal West Pico Chemiluminescent Substrate detection system (Pierce Biotechnology). 


\section{Immunofluorescence}

After transfection and incubation on glass coverslides for 24 hours at $37{ }^{\circ} \mathrm{C}$ and $4.7 \% \mathrm{CO}_{2}$, HeLa cells were washed once with $1 \mathrm{X}$ phosphate-buffered solution (PBS), fixed with Cytofix/Cytoperm (BD Biosciences, San Diego, CA), and blocked for 30 minutes with $1 \mathrm{X}$ Perm/Wash (BD Biosciences). Cells were incubated with primary and secondary antibodies in $1 \mathrm{X} \mathrm{Perm} /$ Wash at $37^{\circ} \mathrm{C}$ for one hour each. ERG-1/2/3 rabbit polyclonal IgG (Santa Cruz Biotechnology) was used as a primary antibody $(0.2 \mu \mathrm{g} / \mathrm{mL})$, Alexa Fluor 488 donkey anti-rabbit IgG was used as a secondary antibody $(0.2 \mu \mathrm{g} / \mathrm{mL}$; Molecular Probes, Eugene, OR), and bis-benzamide ( $1 \mu \mathrm{g} / \mathrm{mL}$, Sigma-Aldrich) was used to stain the nucleus. Coverslips were mounted on glass slides using aqueous mounting media containing anti-fading reagent (Biomeda Corp., Foster City, CA). Slides were examined with the use of a Nikon Eclipse E800 microscope. Z-stack images were obtained using an RT Slider Spot camera (Diagnostic Instruments, Inc., Sterling Heights, MI) and analyzed with Image-Pro Plus, Version 6.2.0.424 (Media Cybernetics, Bethesda, MD) and Autoquant X, Version X2.0.0 (Media Cybernetics) softwares.

\section{Bioinformatic prediction of three-dimensional structures}

Three-dimensional structures corresponding to sequences of the full-length ERG, the truncated ERG, and the functional domains were predicted by using bioinformatic softwares. The MUSTER database was used to generate the threedimensional models of the proteins, ${ }^{26} \mathrm{Jmol}$ was used for visualization (www.jmol.org), and PFAM was used for the domain search. ${ }^{27}$

\section{Analysis of ERG target genes}

Total RNA was purified from transiently transfected HeLa or NIH 3 T3 cells using an RNAqueous-4PCR kit (Ambion, Austin, TX). For cDNA synthesis, $5 \mu \mathrm{g}$ of total RNA was reverse transcribed using random primers (Stratagene, La Jolla, CA) and Superscript II reverse transcriptase (Invitrogen). ERG target genes were detected by PCR using the specifically-designed primers (Invitrogen). The following PCR conditions were used routinely in a Genius thermal cycler (Techne Inc., Burlington, NJ): one cycle, $95^{\circ} \mathrm{C}$, two minutes; 35 cycles, $95^{\circ} \mathrm{C}$, one minute, $60^{\circ} \mathrm{C}$, one minute, $72{ }^{\circ} \mathrm{C}$, one minute; one cycle, $72{ }^{\circ} \mathrm{C}$, five minutes. PCR products were analyzed on a $1 \%$ agarose gel and visualized with ultraviolet light. The amplified bands were quantified using Image-J software.

\section{Results and discussion \\ Cloning and overexpression of the truncated ERG}

The TMPRSS2-ERG gene fusion is heterogeneously expressed due to a variety of interstitial deletions, creating at least 17 distinct transcripts depending on the location of the fusion. Alternative splicing can cause fusions that occur between exons 1, 2, or 3 of TMPRSS 2 and exons 2, 3, 4, 5, or 6 of $E R G$. The most common TMPRSS2-ERG fusion occurs between exon 1 of TMPRSS 2 and exon 4 of $E R G$ (Figure 1C). In this fusion gene, the native ATG is lost and translation is assumed to start from the first ATG. The truncated open reading frame (ORF) was amplified from $\mathrm{VCaP}$ prostate cancer cells and was bidirectionally cloned into expression vector PSG5 as described in Materials and methods (Figure 2A). As expected, for successful ligations between $E R G$ and PSG5, XhoI digests of the clones show two distinct bands, corresponding to the $4.5 \mathrm{~kb}$ vector and the $1.5 \mathrm{~kb}$ insert (Figure 2B, lanes 1 and 2). Upon separate digestion with NheI, plasmids with the insert oriented in the forward direction have one large fragment of approximately $6 \mathrm{~kb}$ and a small undetectable fragment of less than $20 \mathrm{bp}$, which is not detectable on the agarose gel (Figure 2B, lane 3 ). In contrast, plasmids with the insert cloned in the reverse direction should contain fragments of $4.5 \mathrm{~kb}$ and $1.5 \mathrm{~kb}$, which are of similar size to digestion with XhoI (Figure 2B, lane 4). These DNA sequences and orientations were verified by sequencing. Because of their size, plasmids containing the reverse insert served as an ideal negative control in future experiments. The overexpression of the truncated ERG was shown by western blotting (Figure 2C). The molecular weight of the full-length ERG has been estimated to be $49 \mathrm{kD}$. With the deletion of 31 amino acids from the N-terminus, the truncated ERG is expected to be approximately $45 \mathrm{kD}$. Figure $2 \mathrm{C}$ shows that after 36 hours of transient transfection with PSG5-ERG, both NIH3T3 and HeLa cell lines overexpress a protein of approximately $45 \mathrm{kD}$. In contrast, cells transfected with no DNA or with the vector containing a reverse $E R G$ insert exhibit no bands. This indicates that the truncated $E R G$ was successfully cloned and overexpressed in both cells. During the preparation of this manuscript, a published paper verified that the truncated ERG from the TMPRSS2-ERG fusion is indeed translated with the first ATG as an alternative translation initiation site. ${ }^{15}$

\section{Predicted three-dimensional structure of the truncated ERG}

The $E R G$ used in these experiments is modeled after the most common TMPRSS2-ERG fusion which occurs between 
A

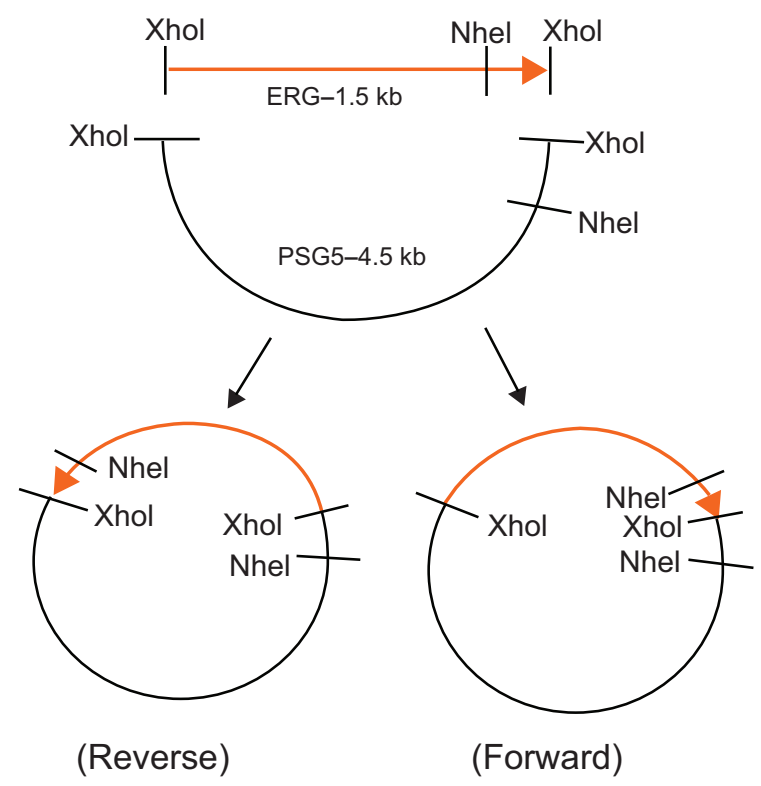

B

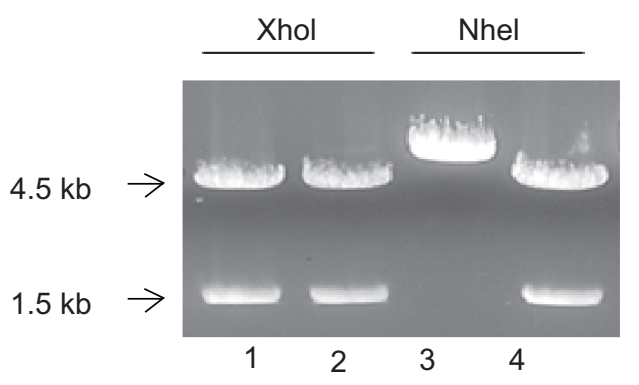

C

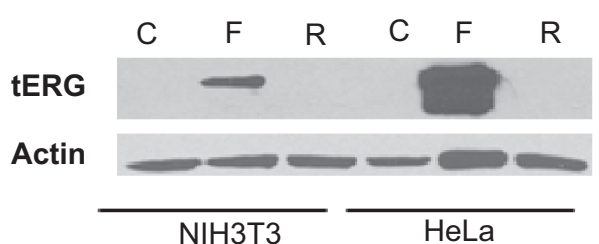

Figure 2 Cloning and overexpression of truncated ERG. A) Schematic strategy in cloning of the truncated ERG into the expression vector PSG5. B) Verification of the insertion by enzyme digestion restriction. Lanes I and 3: Forward clone; and lanes 2 and 4: Reverse clone. C) Overexpression of truncated ERG. C: transfection with no DNA; F: transfected with Forward clone; and R: transfected with Reverse clone. tERG: truncated ERG. The actin serves as internal control.

exon 1 of TMPRSS 2 and exon 4 of ERG. The use of an alternate ATG start codon generates a truncated ERG, which deletes 31 amino acids from the $\mathrm{N}$-terminus of the protein. However, this truncation does not affect the DNA binding domain (ETS) or the integrity of the pointed domain (PTN, Figure 3A), which is particularly important for both dimerization and recruitment of other transcriptional factors. ${ }^{28}$
To determine if the truncation of ERG affects the structure of the protein, we analyzed sequences of both the full-length and truncated ERG with bioinformatic softwares. The predicted three-dimensional structure of the truncated ERG appears to be very similar to that of the full-length protein. Both visibly contain five alpha helices (colored in pink) and multiple beta sheets (shown in yellow, Figure 3B). Although the software

A

\begin{tabular}{|c|c|c|c|}
\hline ADT1 & PNT & ETS & ADT2 \\
\hline
\end{tabular}

B
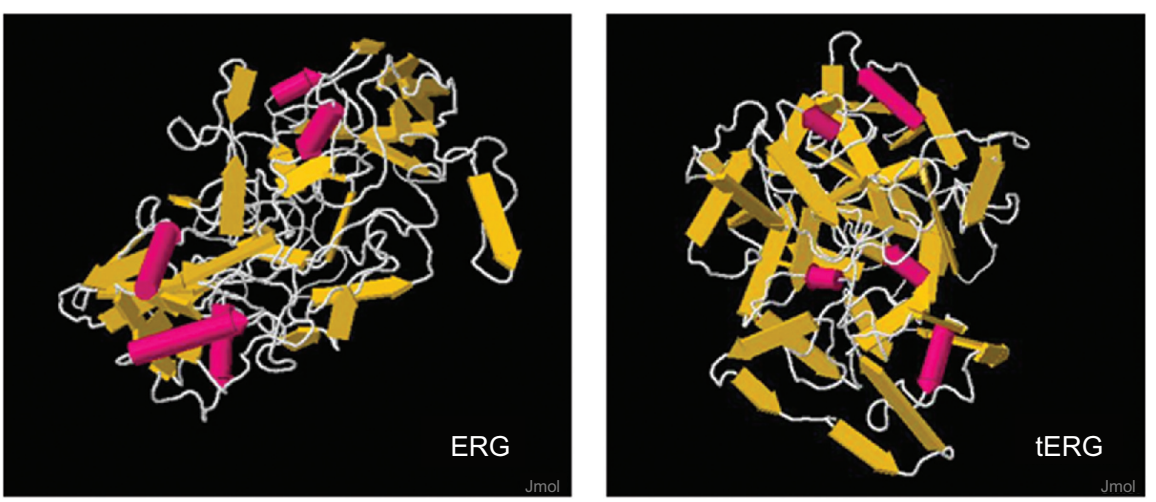

Figure 3 Schematic drawing of the truncated ERG protein A) and modeled three-dimensional protein structure B) of the truncated ERG.The activation domains, ADTI and ADT2, are located in the N- and C-termini, respectively. PNT represents the pointed domain and ETS represents the ETS-binding site. The gray box depicts the $3 \mathrm{I}$ amino acid deletion from the $\mathrm{N}$-terminus. Predicted three-dimensional models of the protein structures of the full-length ERG and truncated ERG show that the overall structure of the truncated ERG appears to be similar to that of the full length. The alpha helices are shown in pink and the beta sheets were in yellow. 
has limited capabilities and could not specifically identify the location of the deletion, the overall structures of the native ERG and the truncated ERG are similar. This suggests that the small truncation in the ORF of ERG does not affect the overall protein structure significantly.

\section{Overexpressed truncated ERG protein localizes to the nucleus}

Since ERG is a transcription factor that binds to the promoter regions of target genes, its function depends on its ability to locate to the nucleus. The nuclear translocation signal sequence has not been identified for ERG. We cannot completely exclude the possibility that it is located in the deleted N-terminus. Therefore, it is important to verify that the nucleus translocation is not affected by the deletion. Immunofluorescent microscopy was performed on HeLa cells transiently transfected with no $E R G$ or with plasmids containing either the forward or reverse $E R G$ insert. Threedimensional images show that cells transfected with the forward insert exhibit intense green staining intermingled with blue nuclear staining, signifying that the protein is not simply residing in cytosolic areas around the nucleus (Figure 4, middle panel). This indicates that the truncated ERG is located in the nucleus. Control cells transfected with no $E R G$ (Figure 4, left panel) or with the reverse insert (Figure 4, right panel) do not express ERG in their nuclei, as evidenced by the lack of green staining. Since cells transfected with PSG5-ERG demonstrate no signs of impaired ERG nuclear localization, this suggests that the small truncation in the ERG does not have a significant effect on subcellular location.

\section{Overexpression of truncated ERG} and regulation of target gene expression

Although the cellular functions of ERG have been established, the genes as well as the genetic pathways regulated by the TMPRSS2-ERG gene fusion product have not been characterized. However, because ERG is a pivotal transcription factor, it is reasonable to speculate that its actions are mediated through the regulation of its target genes. It is expected that the TMPRSS2-ERG gene fusion will first induce ERG overexpression and the overexpressed ERG will
No DNA
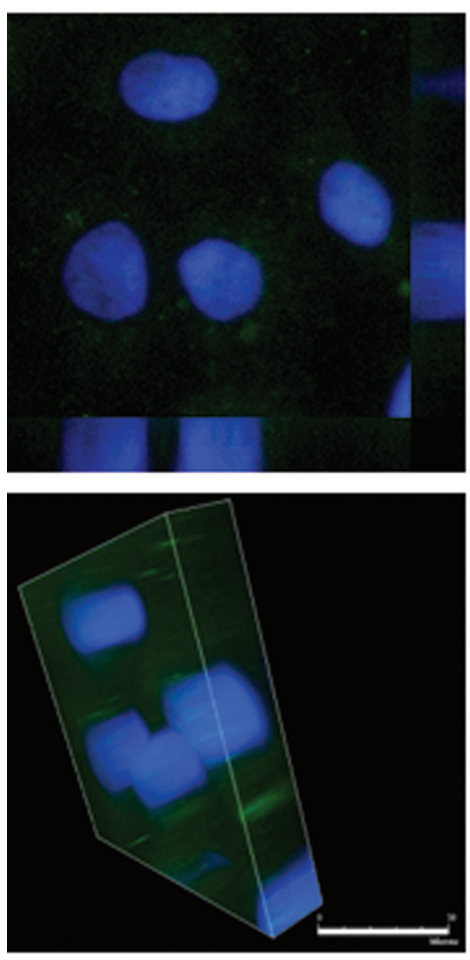

Forward
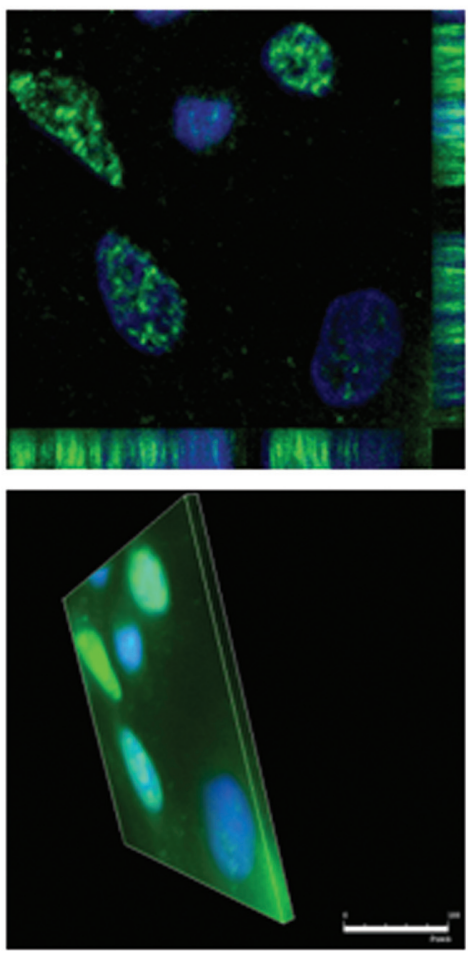

Reverse
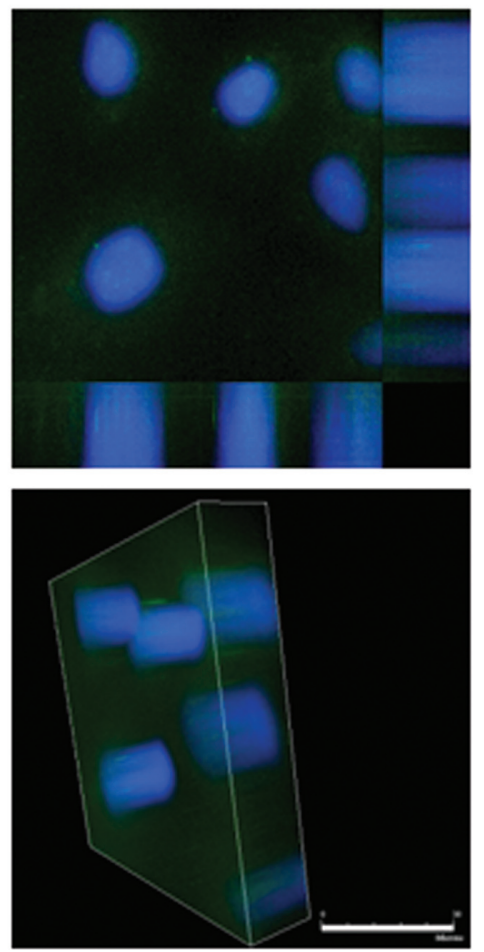

Figure 4 ERG is expressed in nuclei of cells transfected with PSG5-ERG. HeLa cells transfected with plasmids containing the forward insert, the reverse insert, or no DNA were fixed, permeabilized, and stained for ERG (Green) using ERG-I/2/3 rabbit polyclonal IgG as the primary antibody and Alexa Fluor 488 donkey anti-rabbit lgG as the secondary antibody. Bis-benzamide (Blue) was used for nuclear staining. Images are taken with a 60X objective. The top and bottom panels are top- and side-views, respectively. 
then affect the expression of its target genes either through activation or repression. Ultimately, it is the combinations of the target gene products that will lead to prostate cancer development. Previous literature has found that there is a wide variety of target genes that are up- or downregulated by ERG. Through comparison of the ERG-positive and ERG-negative prostate cancers, Iljin and colleagues identified a wide array of genes up- or downregulated by ERG. ${ }^{20}$ Based on the microarray data, a broad screening of seven ERG target genes was first conducted by reverse transcriptase-polymerase chain reaction (RT-PCR) using specifically designed primers (Table 1). It was initially determined that three genes, $A G T$, GPR116, KHDRBS3, appear to be upregulated, whilst the $R A M P 1$ is downregulated in certain degrees by ERG overexpression in both HeLa and NIH3T3 cells. Our results are consistent with the previous findings. ${ }^{20}$ However, the $A G T$ appears to be affected the most. Figure 5 showed that the mRNA level of $A G T$ is significantly upregulated in cells transfected with the forward insert. In contrast, cells transfected with the reverse insert do not exhibit this effect and express levels comparable to those of control cells transfected with no DNA. GAPDH, a housekeeping protein, was used as a loading control.

We speculate that aberrant expression of the truncated ERG could enhance prostate cancer angiogenesis through upregulating AGT expression. The human $A G T$ gene, located on chromosome 1, consists of 5 exons and 4 introns and encodes for angiotensinogen. Angiotensinogen (AGT) is a 452-amino acid glycoprotein that is synthesized and secreted mainly by the liver, but also by the brain. ${ }^{29,30}$ So far, it is the only known precursor of angiotensin I (Agt I), which is produced when AGT is cleaved by renin, an enzyme secreted by the kidneys. Agt I is a decapeptide which remains inactive, with no known function except to be converted by angiotensin-converting enzyme (ACE) into its active form, angiotensin II (Agt II). Agt II plays a major role in the renin-angiotensin system (RAS) and acts as a hormone through its interactions with its specific angiotensin receptors, $\mathrm{AT}_{1}$ and $\mathrm{AT}_{2} \cdot{ }^{31}$ Renin, $\mathrm{AGT}, \mathrm{ACE}$, Agt II, and the angiotensin receptors have been found in the human prostate, prostate cancer cell lines, and rat prostatic cells..$^{32,33}$ The presence of Agt II has been observed in basal cells of the normal prostate, as well as in proliferating neoplastic cells in high grade prostatic intraepithelial neoplasia and malignant prostate cancer cells. ${ }^{34,35} \mathrm{Agt}$ II has been shown to increase the rate of proliferation of prostate cancer cells and it may act as an early marker of malignancy. ${ }^{32,33,35}$ It has been suggested that Agt II is a cell proliferative factor due to the fact that blocking its receptors has been shown to have anti-proliferative activity, as well as roles in decreasing prostate-specific antigen and improving the statuses of patients with hormone-refractory prostate cancer. ${ }^{34,35}$ Other research has provided evidence that Agt II induces oxidative stress, which may lead to prostate cancer. ${ }^{36}$ Chronic inflammation has long been associated with the development of cancer, by releasing reactive oxygen species that cause permanent DNA damage and genomic alterations, particularly in proliferating epithelium. ${ }^{37-39}$ Agt II is involved in a variety of signaling pathways and

Table I Primers used in reverse transcriptase-polymerase chain reaction

\begin{tabular}{|c|c|c|}
\hline Name of the genes & Abbreviations & Primer sequences \\
\hline \multirow[t]{2}{*}{ Angiotensinogen } & AGT & Upper: 5'-CTCTCTGGACTTCACAGAACTGGA-3' \\
\hline & & Lower: 5'-GGGAGTTTTGCTGGAAAGTGAGAC-3' \\
\hline \multirow{2}{*}{$\mathrm{Ca}^{2+}$-dependent secretion activator } & CADPS & Upper: 5'-TACTATCTTGGAAGGAGTGCTGGC-3' \\
\hline & & Lower: 5'-TTCACTCACTGATGCTGTGGCTTC-3' \\
\hline $\mathrm{Ca}^{2+}$-dependent activator protein & CADPS2 & Upper: 5'-CAGTGACGATGAACAAGACCGCAT-3' \\
\hline for secretion 2 & & Lower: 5'-ACTCCATTTCGGGCGCAATACTC-3' \\
\hline \multirow[t]{2}{*}{ G protein-coupled receptor 116} & GPRII6 & Upper: 5'-GGGAGTGGAATGGAACCTATCACT-3' \\
\hline & & Lower: 5'-GGCATGTGATGTTTTCCCCAGGAA-3' \\
\hline KH domain containing, binding, & KHDRBS3 & Upper: 5'-CTGCGCCTGGTGAACCAAGAAATA-3' \\
\hline signal transduction associated 3 & & Lower: 5' - ATAAGCTTCTGCAGGTGGGGCAAA-3' \\
\hline Phospholipase A2, group VII & $2 \mathrm{G} 7$ & Upper: 5'-AAGTACTGATGGCTGCTGCAAGCT-3' \\
\hline (platelet-activating factor & & Lower: 5'-CATGAGATGCCAGGTCAATGCCAA-3' \\
\hline \multicolumn{3}{|l|}{ acetylhydrolase, plasma) } \\
\hline Receptor activity modifying & RAMPI & Upper: 5'-ATCACCTCTTCATGACCACTGCC-3' \\
\hline protein I & & Lower: 5'-TACACAATGCCCTCAGTGCGCTT-3' \\
\hline Glyceraldehyde-3-phosphate & GAPDH & Upper: 5'-ACAGCCTCAAGATCATCAGCAA-3' \\
\hline dehydrogenase (control) & & Lower: 5'-ACCACTGACACGTTGGCAGT-3' \\
\hline
\end{tabular}


A

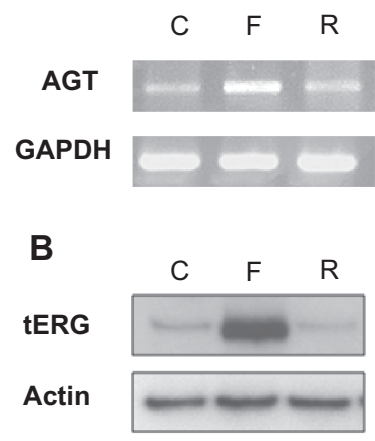

C

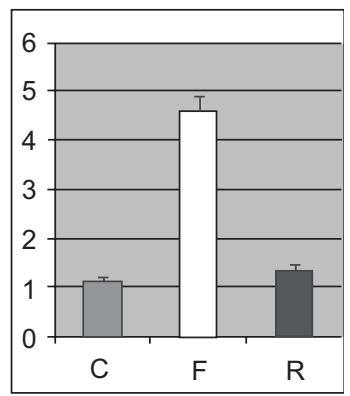

Figure 5 ERG overexpression and regulation target gene expression. A) Total RNA was purified from transfected HeLa cells and CDNA was created. ERG target gene AGT was detected by RT-PCR. GAPDH was used as a loading control. B) Western blot to show the levels of the overexpressed truncated ERG. Actin serves an internal control. C) Histogram of the AGT expression regulated by ERG overexpression. The intensities of the amplified band were quantified using Image-J software. The mRNA levels were normalized with the internal control GAPDH. C: transfection without DNA; F: transfected with Forward clone; and $\mathbf{R}$ : transfected with reverse clone.

it has been shown to stimulate the production of reactive oxygen species by upregulating $\mathrm{NAD}^{+} / \mathrm{NADP}^{+}(\mathrm{NADPH})$ oxidases. ${ }^{34,35}$ Additional studies have demonstrated that there are increased levels of reactive oxygen species-related enzymes and hydrogen peroxide in prostatic epithelial neoplasia and cancer. ${ }^{40}$

In summary, we have successfully cloned and overexpressed the truncated ERG corresponding to the product of TMPRSS2-ERG fusion in both NIH3T3 and HeLa cells. Three dimensional modeling shows that the small $\mathrm{N}$-terminus deletion has little effect in the overall structure. Immunostaining demonstrated that the overexpressed truncated ERG takes a nuclear location. More importantly, overexpression of truncated ERG leads to the upregulation of its target gene $A G T$. Lines of evidence from previous research suggest that AGT plays some roles in prostate cancer development. We speculate that one of the mechanisms for the overexpression of the truncated ERG from TMPRSS2$E R G$ fusion is enhancing angiogenesis through upregulation of angiotensinogen.

\section{Acknowledgments}

The authors want to thank Dr Jiemin Wong (Baylor College of Medicine, Houston, TX) for his kindness in offering the PAS-enhance-Luc reporter and the pOZ-N vector. We are grateful to Dr Ruth Borghaei (Philadelphia College of Osteopathic Medicine, Philadelphia, PA, USA) for her critical reading of the manuscript and valuable suggestions. This work is supported partially by 2006 Formula Grant from Pennsylvania Department of Health, Bureau

of Health Statistics and Research; partially by Center for Chronic Disorders of Aging, Philadelphia College of Osteopathic Medicine, Philadelphia, PA, USA, and partially by the Christian R. Mary F. Lindback Foundation, Philadelphia, PA. USA. The authors report no conflicts of interest in this work.

\section{References}

1. Vindrieux D, Escobar P, Lazennec G. Emerging roles of chemokines in prostate cancer. Endocr Relat Cancer. 2009;16(3):663-673.

2. Greenlee RT, Hill-Harmon MB, Murray T, Thun M. Cancer statistics, 2001. CA Cancer J Clin. 2001;51(1):15-36.

3. Jemal A, Murray T, Ward E, et al. Cancer statistics, 2005. CA Cancer J Clin. 2005;55(1):10-30.

4. Jemal A, Tiwari RC, Murray T, et al. Cancer statistics, 2004. CA Cancer J Clin. 2004;54(1):8-29.

5. Kumar-Sinha C, Tomlins SA, Chinnaiyan AM. Recurrent gene fusions in prostate cancer. Nat Rev Cancer. 2008;8(7):497-511.

6. Johns LE, Houlston RS. A systematic review and meta-analysis of familial prostate cancer risk. BJU Int. 2003;91(9):789-794.

7. Harden J. Developmental life stage and couples' experiences with prostate cancer: a review of the literature. Cancer Nurs. 2005;28(2): 85-98.

8. Denis LJ, Griffiths K. Endocrine treatment in prostate cancer. Semin Surg Oncol. 2000;18(1):52-74.

9. Bostwick DG. Prostatic intraepithelial neoplasia (PIN): current concepts. J Cell Biochem Suppl. 1992;16H:10-19.

10. Tomlins SA, Rhodes DR, Perner S, et al. Recurrent fusion of TMPRSS2 and ETS transcription factor genes in prostate cancer. Science. 2005;310(5748):644-648.

11. Clark JP, Cooper CS. ETS gene fusions in prostate cancer. Nat Rev Urol. 2009;6(8):429-439.

12. Han B, Mehra R, Dhanasekaran SM, et al. A fluorescence in situ hybridization screen for E26 transformation-specific aberrations: identification of DDX5-ETV4 fusion protein in prostate cancer. Cancer Res. 2008;68(18):7629-7637

13. Marx J. Medicine. Fused genes may help explain the origins of prostate cancer. Science. 2005;310(5748):603

14. Tomlins SA, Laxman B, Dhanasekaran SM, et al. Distinct classes of chromosomal rearrangements create oncogenic ETS gene fusions in prostate cancer. Nature. 2007;448(7153):595-599.

15. King JC, Xu J, Wongvipat J, et al. Cooperativity of TMPRSS2-ERG with PI3-kinase pathway activation in prostate oncogenesis. Nat Genet. 2009;41(5):524-526.

16. Carver BS, Tran J, Gopalan A, et al. Aberrant ERG expression cooperates with loss of PTEN to promote cancer progression in the prostate. Nat Genet. 2009;41(5):619-624.

17. Cerveira N, Ribeiro FR, Peixoto A, et al. TMPRSS2-ERG gene fusion causing ERG overexpression precedes chromosome copy number changes in prostate carcinomas and paired HGPIN lesions. Neoplasia. 2006;8(10):826-832.

18. Demichelis F, Fall K, Perner S, et al. TMPRSS2:ERG gene fusion associated with lethal prostate cancer in a watchful waiting cohort. Oncogene. 2007;26(31):4596-4599.

19. Wang J, Cai Y, Ren C, Ittmann M. Expression of variant TMPRSS2/ $E R G$ fusion messenger RNAs is associated with aggressive prostate cancer. Cancer Res. 2006;66(17):8347-8351.

20. Iljin K, Wolf M, Edgren $\mathrm{H}$, et al. TMPRSS2 fusions with oncogenic ETS factors in prostate cancer involve unbalanced genomic rearrangements and are associated with HDAC1 and epigenetic reprogramming. Cancer Res. 2006;66(21):10242-10246.

21. Lucas JM, True L, Hawley S, et al. The androgen-regulated type II serine protease TMPRSS2 is differentially expressed and mislocalized in prostate adenocarcinoma. J Pathol. 2008;215(2):118-125. 
22. Vaarala MH, Porvari K, Kyllonen A, Lukkarinen O, Vihko P. The TMPRSS2 gene encoding transmembrane serine protease is overexpressed in a majority of prostate cancer patients: detection of mutated TMPRSS2 form in a case of aggressive disease. Int J Cancer. 2001;94(5):705-710.

23. Lin B, Ferguson C, White JT, et al. Prostate-localized and androgenregulated expression of the membrane-bound serine protease TMPRSS2. Cancer Res. 1999;59(17):4180-4184.

24. Agoulnik IU, Weigel NL. Androgen receptor action in hormonedependent and recurrent prostate cancer. J Cell Biochem. 2006;99(2): 362-372.

25. Carver BS, Tran J, Chen Z, et al. ETS rearrangements and prostate cancer initiation. Nature. 2009;457(7231):E1; discussion E2-E3.

26. Wu S, Zhang Y. MUSTER: Improving protein sequence profileprofile alignments by using multiple sources of structure information. Proteins. 2008;72(2):547-556.

27. Bateman A, Coin L, Durbin R, et al. The Pfam protein families database. Nucleic Acids Res. 2004;32(Database issue):D138-D141.

28. Oikawa T, Yamada T. Molecular biology of the Ets family of transcription factors. Gene. 2003;303:11-34.

29. Nasjetti A, Masson GM. Hepatic origin of renin substrate. Can J Physiol Pharmacol. 1971;49(10):931-932.

30. Tewksbury DA, Dart RA, Travis J. The amino terminal amino acid sequence of human angiotensinogen. Biochem Biophys Res Commun. 1981;99(4):1311-1315.

31. de Gasparo M, Catt KJ, Inagami T, Wright JW, Unger T. International union of pharmacology. XXIII. The angiotensin II receptors. Pharmacol Rev. 2000;52(3):415-472.
32. Dinh DT, Frauman AG, Somers GR, et al. Evidence for activation of the renin-angiotensin system in the human prostate: increased angiotensin II and reduced AT(1) receptor expression in benign prostatic hyperplasia. J Pathol. 2002;196(2):213-219.

33. Nassis L, Frauman AG, Ohishi M, et al. Localization of angiotensinconverting enzyme in the human prostate: pathological expression in benign prostatic hyperplasia. J Pathol. 2001;195(5):571-579.

34. Uemura $H$, Ishiguro $H$, Nagashima $Y$, et al. Antiproliferative activity of angiotensin II receptor blocker through cross-talk between stromal and epithelial prostate cancer cells. Mol Cancer Ther. 2005;4(11): 1699-1709.

35. Uemura $H$, Ishiguro $H$, Nakaigawa $N$, et al. Angiotensin II receptor blocker shows antiproliferative activity in prostate cancer cells: a possibility of tyrosine kinase inhibitor of growth factor. Mol Cancer Ther. 2003;2(11):1139-1147.

36. Uemura H, Ishiguro H, Ishiguro Y, Hoshino K, Takahashi S, Kubota Y. Angiotensin II induces oxidative stress in prostate cancer. Mol Cancer Res. 2008;6(2):250-258.

37. Coussens LM, Werb Z. Inflammation and cancer. Nature. 2002; 420(6917):860-867.

38. Hussain SP, Hofseth LJ, Harris CC. Radical causes of cancer. Nat Rev Cancer. 2003;3(4):276-285.

39. Smith GR, Missailidis S. Cancer, inflammation and the AT1 and AT2 receptors. J Inflamm (Lond). 2004;1(1):3.

40. Lim SD, Sun C, Lambeth JD, et al. Increased Nox1 and hydrogen peroxide in prostate cancer. Prostate. 2005;62(2):200-207.
Pathology and Laboratory Medicine International

\section{Publish your work in this journal}

Pathology and Laboratory Medicine International is a peer-reviewed, open access journal focusing on innovative basic research and translational research related to pathology or human disease. The journal includes original research, updates, case reports, reviews and commentaries on current controversies. The Academic Sponsor

\section{Dovepress}

of this journal is the Chinese American Pathology Association (CAPA). The manuscript management system is completely online and includes a very quick and fair peer-review system. Visit http://www.dovepress.com/testimonials.php to read real quotes from published authors.

Submit your manuscript here: http://www.dovepress.com/pathology-and-laboratory-medicine-international-journal 